\title{
PROGRAMMABLE PHOTONIC CIRCUITS
}

Review paper - formatted final submitted manuscript

Nature, vol. 586, No. 7828

8 October 2020

Wim Bogaerts ${ }^{1,2}$, Daniel Pérez ${ }^{3,4}$, José Capmany ${ }^{3,4}$, David A.B. Miller ${ }^{5}$, Joyce Poon ${ }^{6,7}$, Dirk Englund ${ }^{8}$, Francesco Morichetti ${ }^{9}$, Andrea Melloni ${ }^{9}$

doi: $10.1038 / \mathrm{s} 41586-020-2764-0$

Received: 17 March 2020

Accepted: 10 July 2020

\section{ABSTRACT}

The growing maturity of integrated photonic technology makes it possible to build increasingly large and complex photonic circuits on the surface of a chip. Today, most of these circuits are designed for a specific application. However, the increase in complexity creates an opportunity for a generation of photonic circuits that can be programmed in software for a wide variety of functions through a mesh of on-chip waveguides, tunable beam couplers and optical phase shifters. Here we discuss the state of this emerging technology, not just the recent developments in photonic building blocks and circuit architectures, but also the higher levels in the technology stack for the electronic control and programming strategies. We also cover the various possible applications in linear matrix operations, quantum information processing and microwave photonics and examine how these generic chips can accelerate the development of future photonic circuits by providing a higher-level platform for prototyping novel optical functionalities without the need for custom chip fabrication.

\section{INTRODUCTION}

Recently, photonic integrated circuits (PICs) have become an established and powerful technology that supports many applications [1], [2]. Like electronic integrated circuits, PICs are implemented on the surface of a chip, but they manipulate light instead of electrical signals, using on-chip optical waveguides, beam couplers, electro-optic modulators, photodetectors and lasers. Whereas electronic circuits are good at digital computations, photonics circuits are good at transporting and processing analogue information. Therefore, today PICs are mostly used in fibre-optic communications, but they are also useful in various applications in which light has an important role, such as chemical, biological or spectroscopic sensors, metrology, and classical and quantum information processing. Because most photonic circuits are tailored for one application, they are called application-specific photonic integrated circuits (ASPICs) and, given that the flow of light is essentially fixed, they can be very compact and power-efficient.

Programmable PICs are based on the idea that the flow of light on the chip can be manipulated at run-time, for example, by electrically controlling tunable beam couplers connected by optical waveguides [3]. This way, light is distributed and spatially rerouted under software control. These chips can implement various linear functions by interfering signals along different paths, and they can define programmable wavelength filters [3], which are essential building blocks for communication or sensor applications or for the manipulation of microwave signals in the optical domain [4], [5]. When scaling up such meshes of connected waveguides, the interferences can perform linear optical computations, such as real-time matrix-vector products[6]-[8]. These are essential operations in quantum information processing [9]-[12], neuromorphic computing and artificial intelligence [6], [7], and we are already seeing rapid development of programmable PIC technologies in these applications. As in electronics, programmability makes it possible to (re)configure the functionality at run-time, which lowers the technological and economic barriers for using the circuit and provides a path to upgradability.

In conventional optics, a system with even a few interferometric elements becomes difficult to line up in terms of both space and wavelength. However, we can now fabricate complex interferometric systems on a chip, with architectures and algorithms for programming, stabilization, and control. Some of these systems even allow self-configuration, adapting

${ }^{1}$ Ghent University - IMEC, Department of Information Technology, Gent, Belgium

${ }^{2}$ Center of Nano- and Biophotonics, Ghent University, Belgium

${ }^{3}$ Universitat Politècnica València, ITEAM Research Institute, Valencia, Spain

${ }^{4}$ iPronics, Programmable Photonics, Valencia, Spain

${ }^{5}$ Stanford University, Ginzton Laboratory, Stanford, CA, USA

${ }^{6}$ Max Planck Institute of Microstructure Physics, Halle, Germany

${ }^{7}$ University of Toronto; Edward S. Rogers Department of Electrical and Computer Engineering, Toronto, Ontario, Canada

${ }^{8}$ Research Laboratory of Electronics, Massachusetts Institute of Technology, Cambridge, MA, USA

${ }^{9}$ Politecnico di Milano - Dipartimento di Elettronica, Informazione e Bioingegneria, Milano, Italy 
(a)

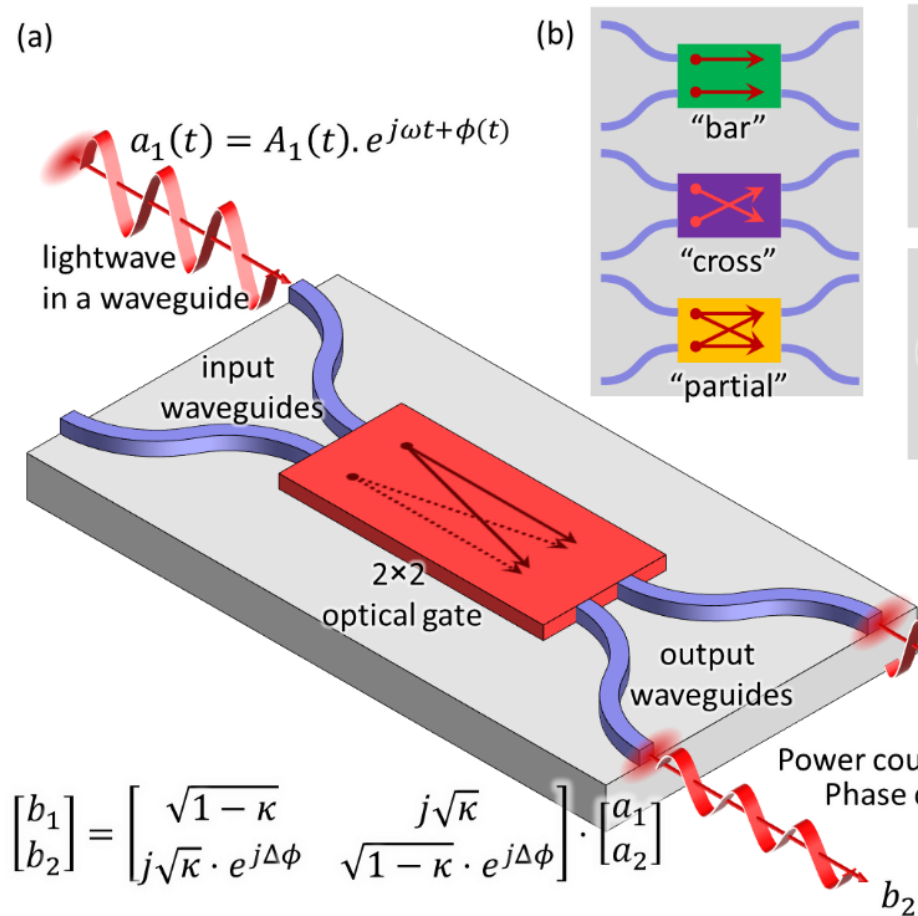

(b)

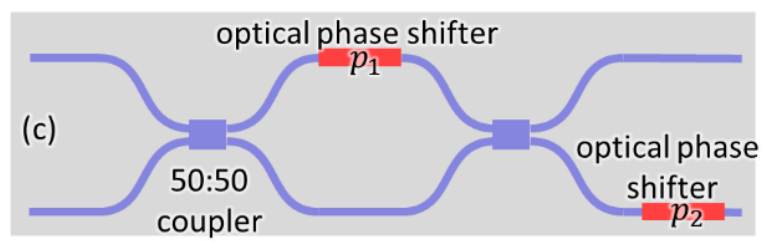

(d)

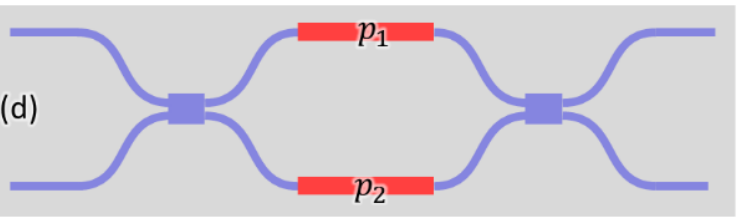

(e)

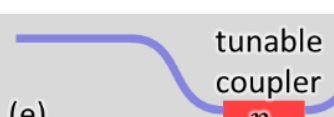
coupler

optical phase
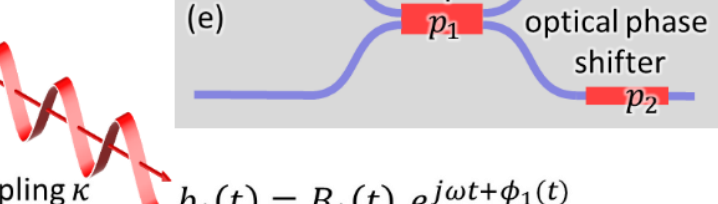

$b_{1}(t)=B_{1}(t) \cdot e^{j \omega t+\phi_{1}(t)}$

$$
b_{2}(t)=B_{2}(t) \cdot e^{j \omega t+\phi_{2}(t)}
$$

Figure 1: Universal $2 \times 2$ optical gates. (a) A $2 \times 2$ optical gate mixes the waves from two input waveguides, controlling both the power coupling and the phase delay. (b) The gate can be tuned between "bar" and "cross" state. (c-e) The gate can be implemented as a circuit with two degrees of freedom $p_{1}$ and $p_{2}$, such as an MZI with two optical phase shifters, or a tunable coupler with an additional phase shifter.

the circuit in real time to the optical problem being solved, without high-level calculations [8], [13], [14]. This combination of complex circuits and control techniques is opening the field of programmable photonics.

Here, we summarize recent developments in this emerging field. We start by explaining the core concepts of waveguide meshes, and how they route light or perform analogue matrix and filtering operations. We then look at the necessary technologies for such photonic circuits. Because programmable PICs are more generic than ASPICs, they can be deployed in various applications, but there are some fields in which their unique capability to perform matrix and parallel operations is especially valuable. With this basis, we look at the future potential of programmable photonics.

\section{MESH ARCHITECTURES AND ALGORITHMS}

In programmable photonic integrated circuits, the flow of light is controlled by waveguides connected in a mesh using $2 \times 2$ blocks, or "analogue gates", the on-chip equivalent of freespace optical beam splitters. The mesh connectivity determines the possible functions of the programmable circuit, and how it can be configured. Some architectures enable arbitrary matrix operations [4]-[6], [8], [12]-[31], and can even automatically adapt to changing problems [8], [13], [14], [29]-[32].

We can separate waveguide meshes into two broad classes: (1) forward-only, where the light flows from one side of the mesh to the other [7], [8], [12], [14], [32], [33]; and (2) recirculating, where light can also be routed in loops and even back to the input ports [3], [5], [18], [23]. Both architectures use the same building blocks: waveguides, $2 \times 2$ couplers, and optical phase shifters that form the analogue optical gates.

\section{THE BASIC BLOCK:}

\section{A $2 \times 2$ ANALOGUE OPTICAL GATE}

A $2 \times 2$ optical gate projects the light from two input waveguides onto two output waveguides as a linear combination, as shown in Figure 1. If all elements are lossless, this corresponds to a unitary transformation. The most common on-chip implementation is a Mach-Zehnder interferometer (MZI), shown in Figure $1 c, d$, and it needs at least two adjustable parameters $p_{1}$ and $p_{2}$ to independently control the power splitting $\kappa$ and the relative phase delay $\Delta \phi$. This can be achieved with two optical phase shifters in different locations within the MZI (Figure 1c,d). When the two fixed couplers in the MZI have a perfect 50:50 split ratio [30], [34], all coupling ratios from $0 \%$ ("bar") to $100 \%$ ("cross") are possible (Figure 1b). An alternative $2 \times 2$ gate combines a controllable coupler and one phase shifter[35] (Figure 1e). These gates work for light flows in both the forward and backward directions. The $2 \times 2$ gate is the key building block for the different mesh architectures we discuss next. 

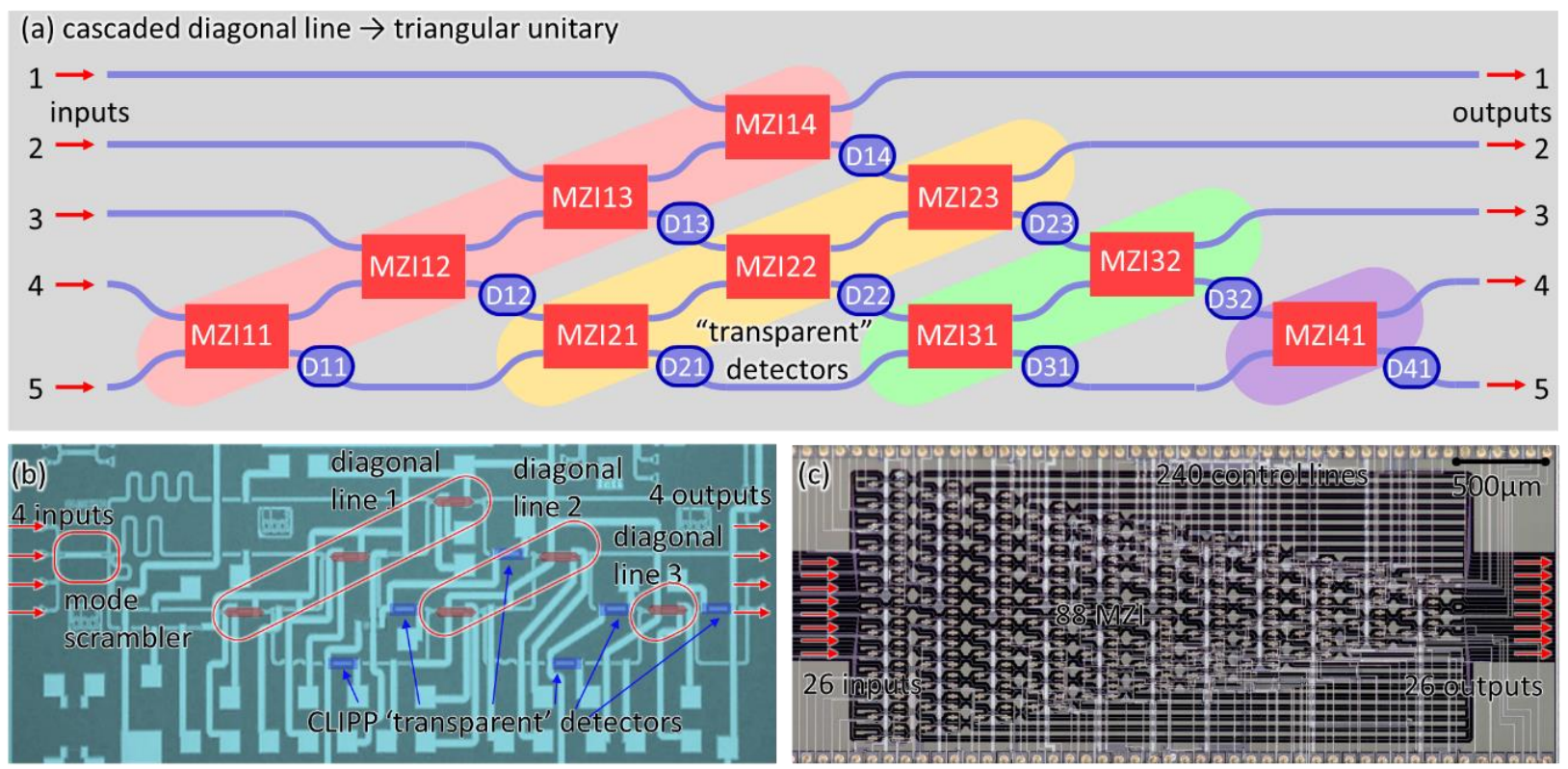

(d) binary tree

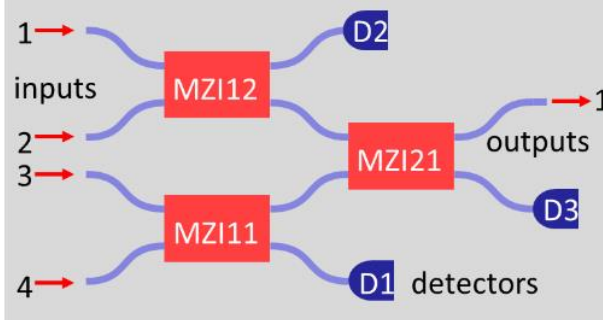

(e) rectangular unitary

Figure 2: Forward-only meshes of $2 \times 2$ optical gates. (a) "Triangular" $5 \times 5$ unitary circuits consisting of 4 "Diagonal lines" of $2 \times 2$ optical gates (MZI11-MZI41) with 'transparent' photodetectors (D11-D41), and one output waveguide. (b) 4-channel "diagonal line" mode "unscrambler" with transparent detectors [31]. (c) Large-scale example with 26 input channels [6], [15] (d) "Binary Tree" self-aligning beam couplers, (e) Rectangular unitary architecture.

\section{FORWARD-ONLY MESHES}

In forward-only mesh networks, light flows in one direction, interfering in $2 \times 2$ gates at every stage. This architecture allows a simple progressive setup - for example, based on minimizing or maximizing power on photodetectors - and in some cases self-configures to specific problems and self-stabilizes their operation [8], [14], [29]-[32], [36].

Figure $2 a$ shows an example of $s$ simple mesh with five waveguides entering a triangular mesh with 'diagonal lines' that can each be configured into a self-aligning beam coupler [29]. When coherent light is injected into the input waveguides of a $2 \times 2$ gate, $p_{1}$ and $p_{2}$ can be sequentially adjusted so that the beams in the lower output waveguide fully cancel out. In Figure 2a we adjust MZI11 to minimize the power onto detector D11, routeing all the light into the upper output waveguide. We repeat this for MZI12, MZI13 and MZI14, thus combining all the light from waveguides $1-5$ into output 1 . This self-configuring algorithm works for any combination of amplitudes and phases in the input waveguides. This algorithm can be run in a repeating loop for real-time adaptation to varying inputs [8], [14], [29], [31].

We can cascade additional "diagonal lines" to perform more complex functions, essentially implementing any linear transformation or matrix between inputs and outputs [8], [29], [33]. The detectors D11 - D14 in Figure 2a need to be almost transparent, passing most of the power to the next 'layer' of the circuit. Each successive 'layer' can then be configured sequentially (see ref [37] for a detailed discussion). Mathematically, amplitude vectors are injected by adjusting the power and phase of the optical modes at the input ports, corresponding to the rows of the desired matrix [30]. Physically, orthogonal input beams could be separated automatically by modulating unique tones on those beams that are then picked up by the corresponding detectors [31].

There are alternative architectures of $2 \times 2$ optical gates. A "binary tree" architecture (Figure 2d) can also be used as an adaptive beam coupler [29], [32]. A rectangular architecture 

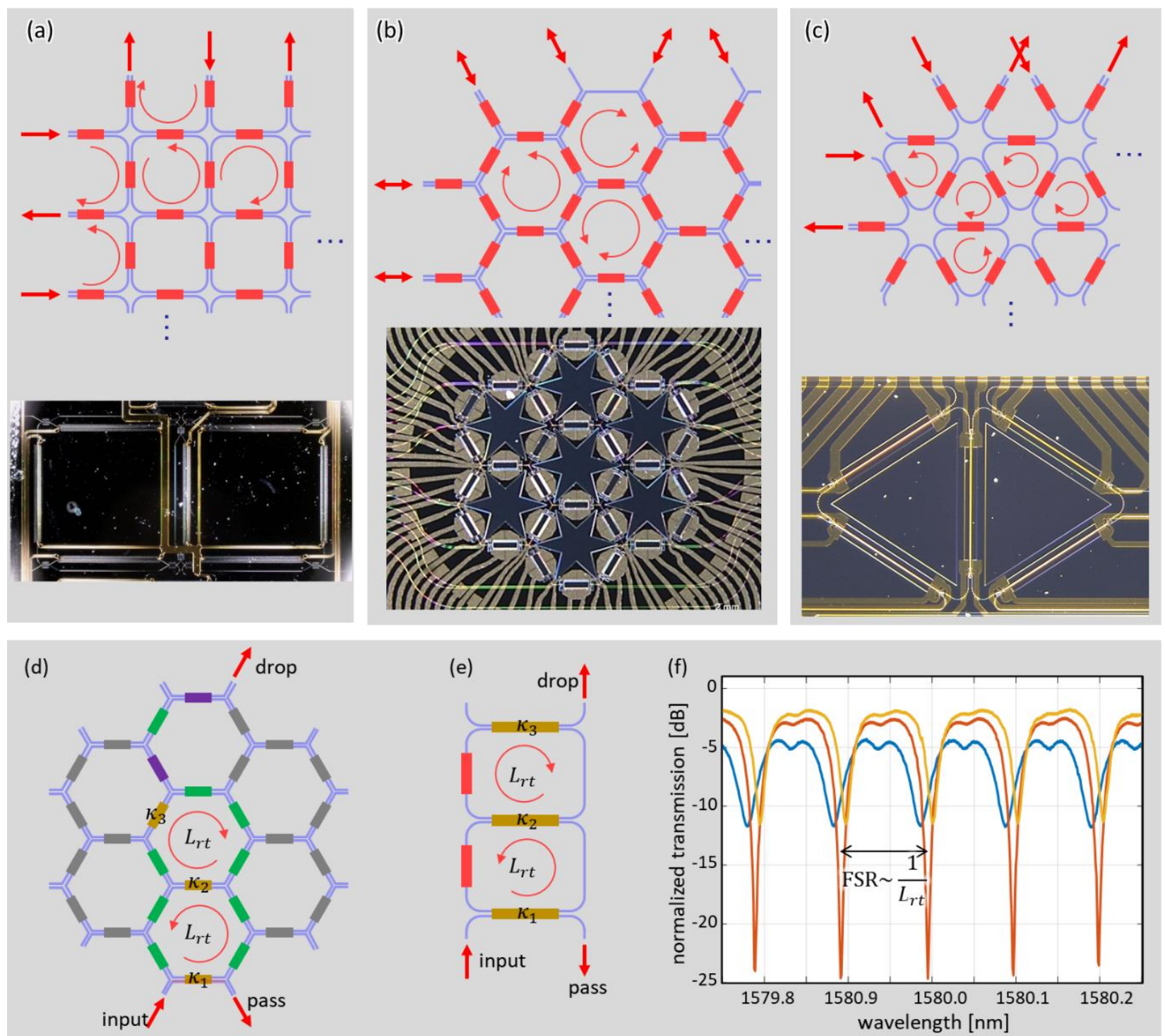

Figure 3: Recirculating waveguide meshes. These can be based on (a) square cells[5], (b) hexagonal cells[44] and (c) triangular cells[35]. (d) Double-ring add/drop wavelength filter programmed in a hexagonal mesh, with (e) the equivalent circuit, and (f) the transmission measurement for different values of the couplings $\kappa_{1}, \kappa_{2}$ and $\kappa_{3}$.

[12], such as the one in Figure 2e, also implements an arbitrary unitary matrix, and there are other architectures which we will not cover in this Review [38]-[40]. Although more compact than a triangular one, the rectangular architecture does not support the convenient self-configuration, but progressive configuration is still possible with embedded detectors [32], [41]. Otherwise, additional optical circuits are needed to configure or calibrate the circuit [32], [42], [43]. For any of these meshes, once the circuit elements are calibrated, arbitrary settings can be programmed directly.

\section{RECIRCULATING MESHES}

Recirculating meshes consist of waveguide loops coupled by $2 \times 2$ optical gates, forming a regular two-dimensional (2D) grid. The loops can route light in any direction through the mesh, allowing us to program a full scattering matrix between all the waveguide ports. Compared to forward-only meshes, these meshes allow delays of discrete lengths (whole number of segments) to implement interferometric and resonant wavelength filters [5], [44].

The loops can be connected in different topologies including square [5], hexagonal [44], triangular [35] and alternative geometries [23], as shown in Figure 3. When evaluating these topologies [3] against integration metrics (such as footprint or gates per area) or functional metrics (such as reconfiguration capability, losses, and the choice of filter periodicity) a hexagonal mesh is particularly attractive, especially because all ports can be used as input or output interchangeably. The mesh can be configured as a forward-only mesh (although with more gates), or it can be programmed as both a finite- and an infinite- 
impulse-response wavelength filter [44]. The number of unit cells in the mesh determines the number and type of functions that can be programmed. A larger mesh is more flexible, but also induces higher optical losses. Figure $3 \mathrm{~d}$-f illustrates a twocavity ring filter in a 7-cell hexagonal mesh. The Free Spectral Range (FSR: the frequency spacing between two resonances) is inversely proportional to the optical roundtrip length, so we want the rings to be as small as possible. As an example: when each of the 'segments' in the mesh has an optical path length of $\sim 1 \mathrm{~mm}$, the largest FSR possible in a hexagonal mesh would be $\sim 50 \mathrm{GHz}$, which suits radiofrequency applications.

Because of the feedback loops, recirculating meshes cannot always be self-configuring (i.e., adjusting themselves without external calculations), but they can be configured through precharacterization or optimization methods [13].

\section{GENERIC PROGRAMMABLE PHOTONICS}

Both forward-only and recirculating waveguide meshes can form the core of a generic programmable photonic circuit as in Figure 4a. These structures are analogous to a fieldprogrammable gate array (FPGA) in electronics [3], [45]. Besides the core, a programmable PIC needs a set of input/output optical signal ports. Additionally, we foresee the introduction of dedicated high-performance blocks to perform specialized (electro-)optical functions such as light sources, high-speed modulators, detectors, optical amplifiers, long delay lines, and high-quality filters, depending on the application. The core waveguide mesh will then interconnect those blocks and will be able to be programmed to emulate some of those functions, such as delay lines or wavelength filters, but not necessarily at the same performance level as dedicated blocks.

\section{THE TECHNOLOGY STACK}

In practice, to make large waveguide meshes work, we also need driver and monitor electronics, control loops and configuration software, as well as conveniently packaged optical and microwave interfaces - all of which are supported by a development kit for engineers who want to integrate a programmable PIC. This technology stack (Figure 4) is similar to that of programmable electronics, but with additional photonic functions.

\section{PIC TECHNOLOGY}

The heart of the programmable circuit is the photonic chip, which can be fabricated in a variety of technology platforms [1], [2], [46], [47]. To accommodate a large number of building blocks, these need to be compact [48], so the most promising platforms are those that have a high refractive index contrast, such as silicon photonics [1], [46], silicon nitride photonics [47] and indium phosphide PICs[2]. These technologies rely on wafer-scale manufacturing similar to electronic fabrication processes.

The optical waveguide losses are of the order of $0.1-1 \mathrm{~dB} / \mathrm{cm}$, depending on the materials and processing quality, which are systematically improving. This translates into $50 \%$ attenuation over $3-30 \mathrm{~cm}$.

At present, most PIC technologies only support a single layer of optical waveguides, restricting connections to a single plane, and giving inherently 2D circuit architectures. Multilayer threedimensional (3D) architectures can increase circuit complexity, flexibility and performance, and reduce footprint. Demonstrated multilayer Si photonic platforms have added integrated silicon nitride (SiN) [46], [49]-[51] or amorphous silicon layers[52], vertically routeing light using low-loss adiabatic tapers. Such layers allow waveguide crossings with ultra-low-loss and cross-talk[49].

\section{PHASE SHIFTERS/TUNABLE COUPLERS}

The key building blocks - the tunable $2 \times 2$ coupler and and phase shifter as in Figure 1 - require low optical insertion loss and low electrical power consumption. In recirculating meshes, the elements should also have a short optical path length, allowing the synthesis of large FSR filters. Today, most programmable photonic circuits use electrically-driven heaters to induce a thermo-optic phase shift [53], [54]. Such thermal tuners are easy to use, but each of them dissipates several $\mathrm{mW}$ of electrical power (in silicon), has a time constants of $10-100 \mu \mathrm{s}$, and can have thermal crosstalk that needs to be compensated [55]. Faster tuners can use free carriers in semiconductors [56], [57], but they have much higher optical losses; furthermore, their loss depends on the induced phase shift, which destroys the basic 'unitary' function of the gate. Research on improved or alternate approaches to phase shifters is very active. The various approaches include materials with high thermo-optic coefficient [58], piezoactuators [59], [60], liquid crystals [61], [62], Pockels effect in perovskites [63]-[65] or polymers [66], and micro-electromechanical systems (MEMS) [67], [68]. Nonvolatile actuators, which maintain their state without an 'always-on' control signal, are also interesting. Examples include memristors [69], phase change materials [70], [71], or mechanically-latched MEMS [67]. The $2 \times 2$ gates that contain these actuators are the primary source of loss in the circuit, with values of $\sim 0.05-0.2 \mathrm{~dB}$ per gate. Technological improvements and better designs are decreasing these values, as required for scaling up the circuits. 


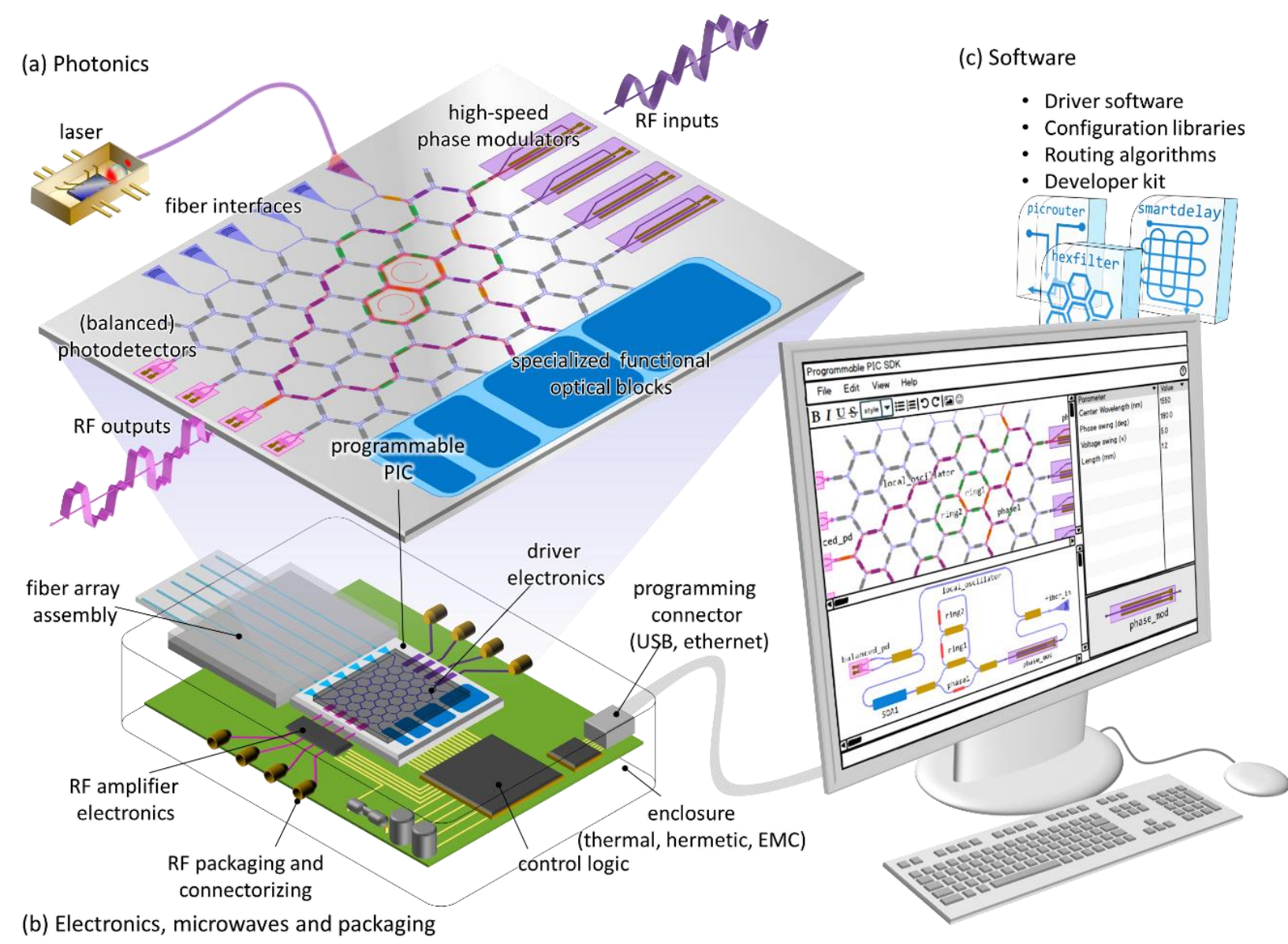

Figure 4: Technology stack for programmable photonic circuits. Programmable photonic integrated circuits require several functional layers. (a) the photonic chip has a programmable mesh of photonic gates, connected to control electronics, optical fibers, and high-speed modulators and detectors to translate between the optical and microwave domains. (b) The photonic chip is packaged together with analog and digital driver electronics. (c) Software algorithms and programming layers give the user access to the photonic functionality.

\section{MONITORS AND CONTROL LOOPS}

With hundreds of thousands of possible optical paths, we need to know where the light is on the chip so we can control the actuators. Photodetectors embedded right after [14], [72] or even inside [73], [74] each tunable element can directly track the path of light [75]. To minimize the optical losses, such monitor photodetectors should be as transparent as possible. Approaches include waveguide taps [14], [76], in-line photodetectors [72], [77], or measuring absorption already present in the waveguides [72]-[74].

With such monitor signals we can operate the circuit as a selfconfiguring beam coupler [14], [29], [74] or implement selfcalibration algorithms [8], [31], [37]. Elementary tunable cells with integrated monitors allow for very simple local control loops that do not need a centralized control system, similar to reflexes in the human nervous system - for example, keeping a $2 \times 2$ gate at a specific working point or stabilizing a filter circuit's wavelength. The feedback loops can be implemented using analogue electronics[75], digital electronics or software [14].
In large programmable PICs, many optical signals can propagate in the same waveguides but on different wavelengths, polarizations or directions. To disentangle these signals in a monitor detector, we can use some form of labelling [8], [31], [36], such as low-amplitude modulation tones at unique frequencies [8], [31]. A dithering tone can also be applied to each actuator, to disentangle their effect on the signal.

Monitors on the edge of the circuit can use non-transparent detectors with higher sensitivity, bandwidth and signal to noise ratio [6], [36], but they provide only limited insight into the internal distribution of light inside the mesh. Forward-only meshes can be fully configured with external monitors, using specific algorithms for continuous adaptation [8], [29], [30], [32], [36], [41]. However, as circuits are scaled up, it becomes harder to control thousands of actuators using a handful of edge detectors. This leads to advanced non-convex global optimization algorithms [7], [11], [13], which require a centralized electronic control system. The optimal trade-off between the number of monitors and the complexity of the control depends on the architecture (for example, forward-only vs. recirculating), and its partitioning.
For the final published text, see nature.com 


\section{ELECTRONICS, RF COMPONENTS AND PACKAGING}

Controlling thousands of actuators and monitors requires a substantial amount of electronics, in a combination of analogue (simple reflex-like control loops) and digital (global control) circuits. The actuators need high-resolution digital-to-analogue converters (DAC) with sufficient precision. Alternatively, we could drive 'slow' actuators with direct digital pulse-width modulation [62], [78]. Likewise, monitor readout requires analogue-to-digital (ADC) conversion circuitry. We also need programmable control logic 'above' such low-level driver electronics; this can rely on microcontrollers, FPGAs or digital signal processors (DSPs), operating on their own or connected to a computer through USB or ethernet.

These electronics need to be physically interfaced with the photonics chip. Photonic-electronic integration on the same chip offers the closest integration [79]-[81], but today such monolithic integration always incurs trade-offs (waveguide losses, limited choice in transistor nodes) that limit scaling to large programmable PICs. For this reason, hybrid integration approaches, where electronics and photonics are fabricated on separate chips, is still the most practical. Traditional wirebonding on the edge of the chip cannot easily scale up to $>1000$ wires, so approaches based on flip-chipping (Figure 4b), interposers or 3D stacking are the most promising long-term solutions [82], [83]. Multiplexing techniques such as rowcolumn addressing of actuators can reduce the number of wires [84].

Photonic waveguides support a massive signal bandwidth, which is why they are used for high-speed communications. To encode high-speed, radio-frequency signals (digital or analogue) onto an optical carrier, we rely on high-speed electro-optic modulators, with bandwidths exceeding $50 \mathrm{GHz}$ [57], [85]. The conversion of modulated optical signals to radiofequency waves is handled by integrated photodetectors [85], [86]. These modulators and photodetectors provide the input and output ports for microwave signals which are then processed on the optical chip [3]-[5], [87]. Such filter functions programmed in the waveguide mesh must have a sufficiently large FSR to capture the entire RF bandwidth, and for this, the optical path lengths in the mesh need to be kept short (for example, an optical 'segment' length of $1 \mathrm{~mm}$ in a hexagonal mesh for a $50 \mathrm{GHz}$ FSR).

High-speed RF interfaces require specialized substrates, connectors, and well-designed stripline waveguides [82], [83], [88]. Since such programmable PICS may also need thousands of electrical control lines and multiple optical fiber connections, packaging is a nontrivial part of the necessary technology stack.

\section{AMPLIFIERS AND LIGHT SOURCES}

The large waveguide meshes of programmable PICs will accumulate higher optical losses than more compact specialized circuits. On-chip amplifiers could compensate such losses, either inside the waveguide mesh or on the edge. When combined with a waveguide cavity, such amplifiers can form a programmable laser, or their nonlinear behavior can enable alloptical signal processing [89].

Not all technology platforms include gain elements. III-V semiconductor platforms allow gain [2], but integration of III-V amplifiers in silicon photonics is challenging; today, most silicon photonic circuits use external light sources. Amplifiers can be bonded on the silicon [90], and recent studies show the longterm potential of direct epitaxy [91]. Techniques like microtransfer printing [92] allow placement of amplifiers in cavities, and bonding on the back side of the silicon-oninsulator wafer [93] gives additional flexibility, allowing arbitrary placement of III-V material relative to Si features.

\section{PROGRAMMING ALGORITHMS AND ROUTEING}

Configuring thousands of actuators for flexible and powerful applications requires programming algorithms and automated methods. Some forward-only meshes can be progressively configured for different linear transformations [29], [37] or layered self-configuring meshes [30], [37]. For overdimensioned meshes with built-in redundancy, where multiple configurations can result in the same functionality, these algorithms need to be generalized.

Recirculating architectures (which are generally overdimensioned) require new layers of programming. Two broad classes of such programming techniques are "black-box" optimization methods [13], [94], [95] (for example machine learning), and synthesis techniques. Optimization methods can operate directly on the hardware and do not necessarily require calibration. Synthesis techniques first deduce the desired mesh configuration and then apply that to the actuators; this requires periodic calibration to assess each element's operating point [94]. Calibration data can be used in the synthesis process itself, for example, to minimize the cost function for routeing light through the mesh [13], [94], [95], or to compose delay lines with a specific dispersion [87].

Programming strategies can also leverage excess capacity to incorporate redundancy and self-healing [3], [95], [96]. Even with imperfect fabrication and defective elements, the mesh architecture and the software layer together can form a reliable system. 

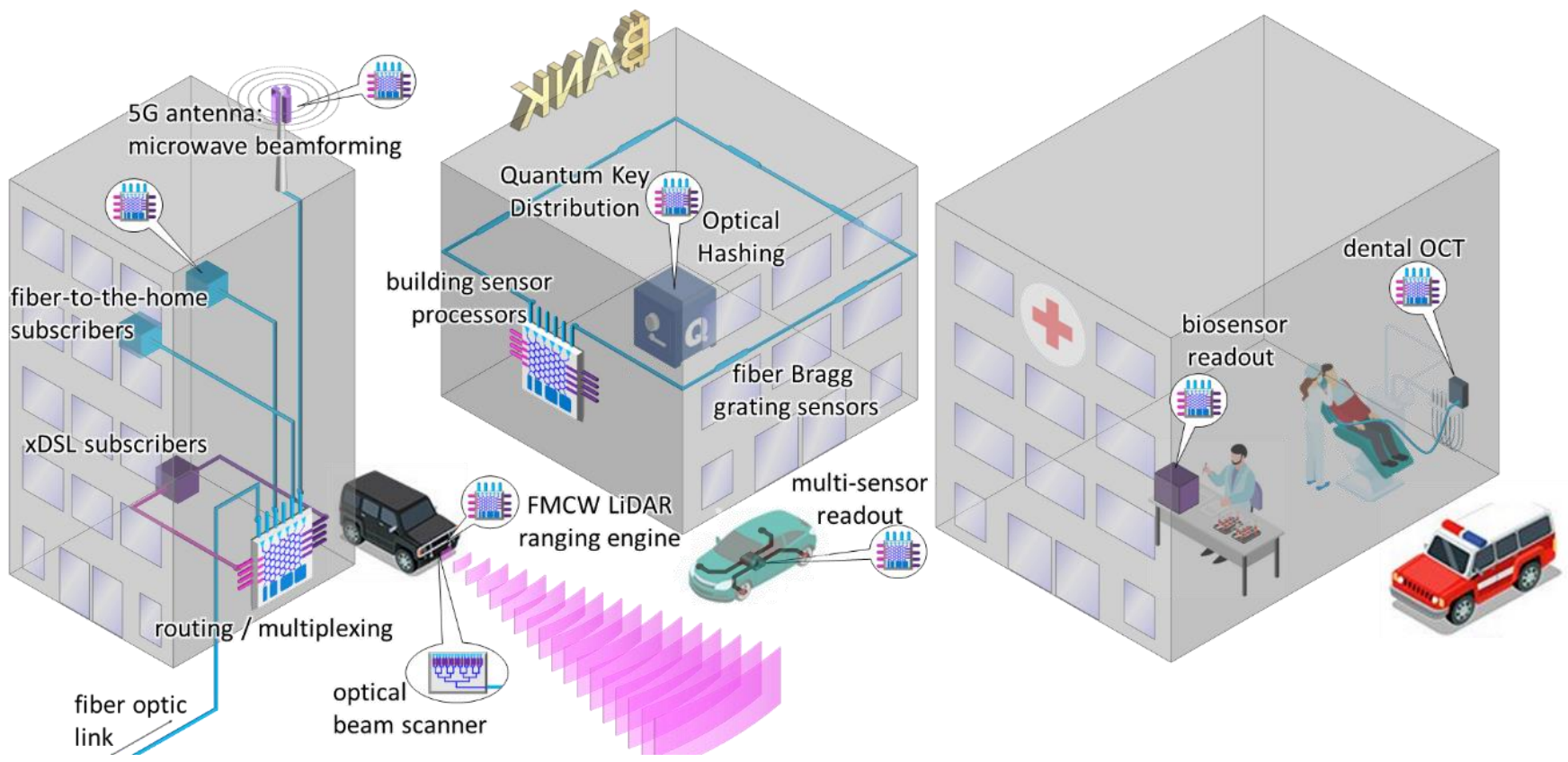

Figure 5: Applications for programmable photonic circuits. Microwave signals can be transported over fiber and processed in the optical domain for XDSL and 5G applications[98]. Optical sensor systems can be used in construction, automotive, security and medical environments, such as for optical coherence tomography (OCT), or frequency-modulated continuous wave (FMCW) LiDAR.

As photonic circuits become programmable, the growing development community will need a programming infrastructure around the photonic+electronic hardware, such as development kits and an application programming interface (API). Just as an electronic FPGA is described in its own language (VHDL), programmable PICs might require their own descriptive language. With high-level programming capabilities comes the possibility of defining reusable routines for operating the programmable PICs. As in electronics, reusable blocks of code can dramatically shorten development time and lead to true photonic/electronic systems-on-chip (SoC).

\section{APPLICATIONS}

Photonic chips differ from electronic chips in their basic operation and in the functions they can efficiently execute. We therefore expect the two types of chips to be complementary: we do not expect to use photonic circuits to perform the digital computations at which electronics excel, but rather for communications, sensing and broadband analogue signal processing.

Programmable photonic chips are naturally more generic and flexible than custom-designed circuits. Of course, this programmability is only useful if these chips can be used in more than one setting [97]. PIC development has mainly been driven by high-speed communication (both telecom and datacenters), but other applications such as sensing and information processing are being explored. As in electronics, we expect different modes in which programmable PICs will fit into the ecosystem:
1. Full-custom ASPICs are today the most commonplace photonic chips. Like electronic application-specific integrated circuits (ASICs), they offer optimum performance for the desired function. But they take a long time to develop, and only make economic sense if the performance or fabrication volume justifies that cost. Still, even in such applications, programmable PICs could accelerate the development in early prototyping. But like FPGAs in electronics, programmable PICs will always have a larger footprint, higher power consumption and higher optical losses than the specialized ASPICs.

2. Combining programmable photonics with ASPICs can lead to hybrid chips where a programmable core is embedded inside a custom ASPIC. Reuse of these cores (and their control logic) can shorten the development time of an ASPIC, just like reusable 'intellectual property' (IP) blocks in complex electronic chips. These hybrid chips have the same manufacturing pipeline as ASPICs, but adding programmability can make them more flexible.

3. Programmable and self-configuring PICs can be used for applications that require adaptation to problems that change in real time, such as adaptive sensing, alignment to changing external optical conditions, and modeunscrambling. In this case, they open up application areas beyond those of fixed ASPICs.

These different scenarios can be found in many application domains, some of which are illustrated in Figure 5. 


\section{QUANTUM INFORMATION PROCESSING AND ARTIFICIAL NEURAL NETWORKS}

Programmable waveguide meshes can be configured to execute any linear transformation between sets of input and output waveguides[8], acting as a matrix operator that performs real-time calculations in the short time that the light needs to traverse the circuit. Such operations are a key function in artificial neural networks, so a programmable PIC can act as an accelerator for artificial intelligence and deep-learning applications[6], [7].

The same linear operations are also at the core of photonic quantum computing. Here, the quantum information is represented by quantum states of light propagating through the PIC [101]. A popular scheme encodes a quantum bit (qubit) as a single photon in a superposition of two waveguides ('rails') [9].

Deep learning and quantum information processing need more functional elements than just the linear circuits: a nonlinear response is needed to implement either a neural 'activation function' or a two-qubit operation [7]. Both deep learning and quantum computing are seeing a rapid co-development of algorithms and hardware. Althought a lack of sufficient error correction still prevents general-purpose quantum computing, noisy intermediate-scale quantum (NISQ) processors[116] have attained 'quantum supremacy', where they outperform classical computers at certain tasks [117]. NISQ devices will now have to prove themselves useful in practical applications. One such scheme is quantum machine learning [118] that promises to process large data sets vastly faster than classical computers.

One way to implement quantum machine learning parallels classical photonic deep neural network accelerators (BoxFigure): stages of linear waveguide meshes connected by activation layers -- except that these activation layers must now have strong coherent (reversible) nonlinearities [119]. In such a 'quantum optical neural network' (QONN), the task of programming an NISQ computer reduces to training the phases in the waveguide mesh through supervised learning on input and output quantum states. The QONN can be taught to perform a range of quantum information processing tasks, including a new approach to quantum optical state compression and reinforcement learning. Recently, a QONN overcame the challenge[120] of programming a one-way quantum repeater [119].

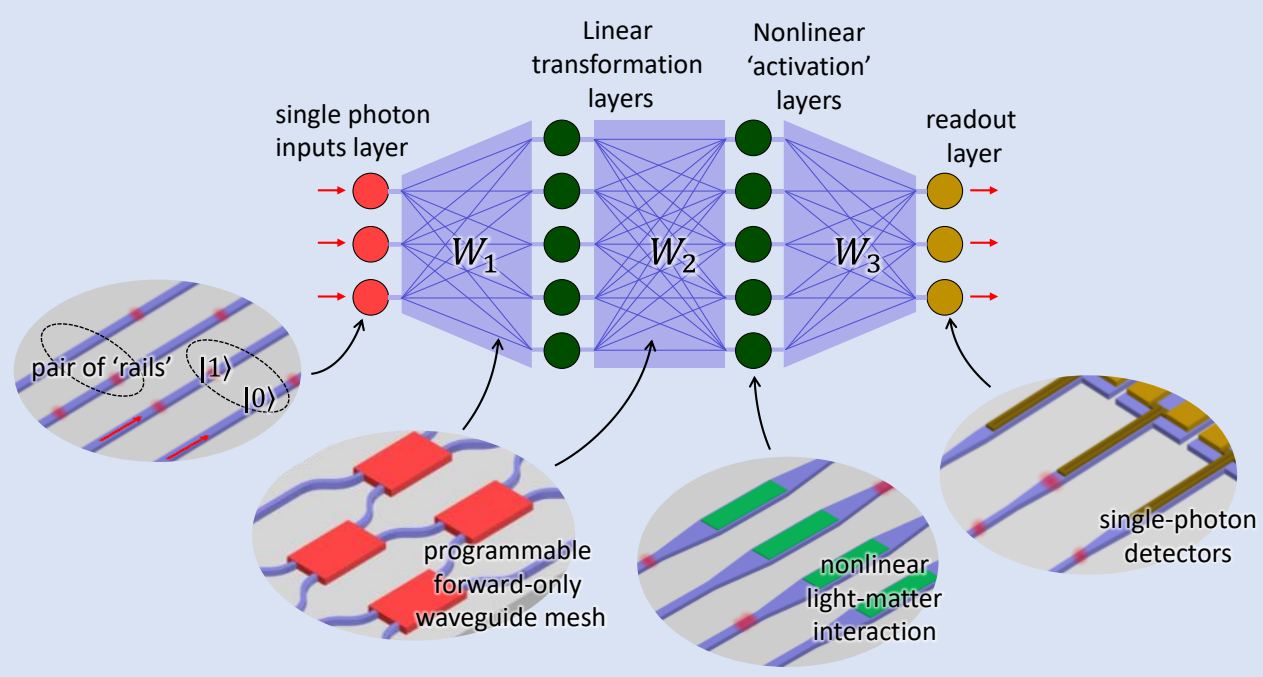

Boxfigure: A quantum optical neural network based on programmable photonics. Such a network is fed by single photons and nonlinear activation (e.g. nonlinear materials or atomic nonlinearities). The final state may be measured to complete a quantum computation or passed into a quantum network.

\section{LINEAR VECTOR-MATRIX PRODUCTS}

The optical field at the output of a reconfigurable waveguide mesh is a linear combination of the signals at the input ports. Such a linear transformation is mathematically described as a multiplication of a vector (the input signals) and a matrix (the mesh), which is executed in real time by propagating light through the circuit [8]. Such matrix operations can be used in signal processing [36], [89] and hashing operations [99], [100], but are also essential in emerging fields like quantum information processing [9], [15], [101] and artificial neural networks [6], [7] (see box).
The matrix can also be used as a switching network in optical communication, as a (de)multiplexer/ (un)scrambler for different modes travelling over an optical fiber [31], or even, when used in pairs, to find the optimal orthogonal communication channels through a linear system [28].

\section{MICROWAVE PHOTONICS}

In microwave photonics, high-frequency electrical signals are processed in the optical domain by modulating them on an optical carrier. Photonic circuits for microwave applications [4] are mostly ASPICs implementing filtering [102], waveform generation [103], reconfigurable delay lines [104] or frequency 
measurements [105]. These functions can all be implemented in generic recirculating waveguide meshes [3], [5]. For instance, the meshes in Figure $3 a$ can be used as a Hilbert transformer, a delay line, a notch or bandpass RF filter, a temporal differentiator or integrator, frequency converter and a programmable delay line [5], [106]. These functions are essential to scale down equipment for next-generation $5 \mathrm{G}$ wireless systems [98] and radiofrequency systems for aerospace, where photonic chips can reduce power consumption and weight. Several use cases for microwave photonics are illustrated in Figure 5.

\section{OPTICAL BEAMFORMING}

Developments in optical beamforming are being driven strongly by LiDAR (light detection and ranging) technology [107]. A beam of light can be constructed using an array of small optical 'antennas' by carefully controlling the phase and amplitude in each antenna [108]. This requires a large number of independent tuners, as in generic programmable circuits. While a generic programmable PIC could serve some simple beamforming applications, the performance requirements (e.g. optical loss, power consumption) will likely dictate the use of dedicated circuits. But optical beamforming can also be used in reverse, coupling a distorted incoming field into a single waveguide [109]. The self-aligning beam coupler [29] can be used in both directions to align itself automatically and adaptively between a source and target [110].

\section{SENSING APPLICATIONS}

Light can be used to sense a wide variety of phenomena through changes in absorption, phase or wavelength response. PICs form an effective sensor platform for transducers (for example, detecting biomolecules [111]), on-chip spectrometers [112], readouts for fiber Bragg gratings, laser doppler vibrometers [113], optical coherence tomography (OCT) and frequency-modulated continuous wave (FMCW) LiDAR receivers. Many of these could be implemented on a generic programmable PIC, and specialized sensor readout circuits could well become a primary application space. Using the generic chip as the transducer is less likely, as the sensing mechanism often requires specialized geometries, chemistry or functionalization on-chip.

\section{PERSPECTIVES}

Programmable PICs have the potential to change the way people use coherent light to manipulate information. Decades ago, programmable electronics went through a similar evolution with microprocessors, FPGAs and DSPs, where it was no longer necessary to design a custom chip to perform a certain function. Instead, an ecosystem arose around off-theshelf programmable electronics, which made it possible to build products much faster, more cheaply and with lower error margins [114]. Photonics is already following the electronics model with foundry-based manufacturing [46], [47]. Programmable PICs, when available on an industrial scale, can take that further, shortening the lead time for photonic chips from months to days, eliminating substantial non-recurrent engineering costs, and shifting product development from hardware to software [97]. This can bring PICs within reach of the Maker community, just as it happened with programmable electronics and additive manufacturing [115]. As in electronics, where discrete elements, ASICs and programmable ICs coexist, we expect a photonic ecosystem where discrete optics, ASPICs and programmable PICs provide their own set of solutions. Although photonic circuits and electronic circuits are both chipbased technologies, they are fundamentally different. One key consideration is scaling: the size of photonic building blocks, and the number integrated on a single chip, is fundamentally limited by the refractive index contrast of the materials. With silicon photonics, we are already approaching the density limit of $\sim 1$ million components per $\mathrm{cm}^{2}$. But there is still a huge space to explore in photonic design complexity.

Photonic circuits and electronic circuits are complementary Because photonic circuits are analogue circuits, programmability in photonics will apply to different functions from those in programmable electronics. This also means that the abstraction layers that have been devised for electronics will need to be reimagined for programmable photonics. This opens up new research fields on top of hardware, and presents opportunities to establish a certain level of standardization, to help designers define and simulate functionality irrespective of the photonic hardware vendor.

\section{SUMMARY}

We have introduced the emerging field of programmable PICs. Today, with application-specific PICs already deployed in a variety of applications, an opportunity arises for generalpurpose programmable PICs. To realize this, a complete technology stack is needed complementing photonics with electronics, packaging and various layers of software. These concepts are already being tested for specialized applications in quantum information processing and neural networks, but general-purpose hardware could be useful for a variety of other applications because its reconfigurability can drastically shorten product development, and its ability to adapt to changing problems offers new possibilities in functional optical systems. 


\section{ACKNOWLEDGEMENTS}

David A.B. Miller acknowledges support from the Air Force Office of Scientific Research (AFOSR) under award number FA9550-17-1-0002.

D. Englund acknowledges support from AFOSR award FA955016-1-0391 and Air Force Research Laboratory award FA8750$16-2-0141$.

Wim Bogaerts acknowledges the European Research Council (ERC) for the support through consolidator grant number 725555 (PhotonicSWARM) and the European H2020 program for the project grant 780283 (MORPHIC).

José Capmany and Daniel Pérez acknowledge the European Research Council (ERC) for the support through advanced grant number 741415 (UMWPCHIP) and Proof of Concept Grant 859927 (FPPAs).

Francesco Morichetti and Andrea Melloni acknowledge the European Commission for the support through H2020 grant number 829116 (Super-Pixels), and Polifab, Politecnico di Milano (www.polifab.polimi.it), for supporting the review of technology aspects.

\section{COMPETING INTERESTS}

The authors declare not competing interests.

\section{REFERENCES}

[1] X. Chen et al., "The Emergence of Silicon Photonics as a Flexible Technology Platform," Proc. IEEE, vol. 106, no. 12, pp. 2101-2116, 2018.

[2] M. Smit, K. Williams, and J. van der Tol, "Past, present, and future of InP-based photonic integration," APL Photonics, vol. 4, no. May, p. 050901, 2019.

[3] J. Capmany and D. Perez, Programmable Integrated Photonics. Oxford University Press, 2020.

The first book on the subject of programmable photonics, giving a detailed overview of the fundamental principles, architectures and potential applications.

[4] D. Marpaung, J. Yao, and J. Capmany, "Integrated microwave photonics," Nat. Photonics, vol. 13, no. 2, pp. 80-90, 2019.

[5] L. Zhuang, C. G. H. Roeloffzen, M. Hoekman, K. Boller, and A. J. Lowery, "Programmable photonic signal processor chip for radiofrequency applications," Optica, vol. 2, no. 10, pp. 1-6, 2015.

[6] Y. Shen et al., "Deep learning with coherent nanophotonic circuits," Nat. Photonics, vol. 11, no. June, pp. 441-447, 2017.

[7] N. C. Harris et al., "Linear programmable nanophotonic processors," Optica, vol. 5, no. 12, pp. 1623-1631, 2018.
One of the largest-scale demonstrations of a programmable photonic circuit, using a silicon photonics forward-only mesh that maps 26 input modes onto 26 output modes, for use in in deep learning and quantum information processing.

[8] D. A. B. Miller, "Self-configuring universal linear optical component," Photonics Res., no. 1, pp. 1-15, 2013.

This foundational paper in the field of programmable photonics is the first to bring together waveguide meshes with self-configuration algorithms that require no active computation, including the concept of the self-aligning beam coupler.

[9] J. Carolan et al., "Universal linear optics," Science, vol. 349, no. 6249, pp. 711-716, 2015.

[10] N. C. Harris et al., "Large-scale quantum photonic circuits in silicon," Nanophotonics, vol. 5, no. 3, pp. 456-468, 2016.

[11] J. Notaros et al., "Programmable dispersion on a photonic integrated circuit for classical and quantum applications," Opt. Express, vol. 25, no. 18, pp. 21275-21285, 2017.

[12] W. R. Clements, P. C. Humphreys, B. J. Metcalf, W. S. Kolthammer, and I. A. Walmsley, "An Optimal Design for Universal Multiport Interferometers," Optica, no. 2, pp. 1-8, 2016.

[13] D. Perez-Lopez, "Programmable Integrated Silicon Photonics Waveguide Meshes: Optimized Designs and Control Algorithms," IEEE J. Sel. Top. Quantum Electron., vol. 26, no. 2, 2020.

[14] A. Ribeiro, A. Ruocco, L. Vanacker, and W. Bogaerts, "Demonstration of a 4x4-port universal linear circuit," Optica, vol. 3, no. 12, pp. 1348-1357, 2016.

[15] N. C. Harris et al., "Quantum transport simulations in a programmable nanophotonic processor," Nat. Photonics, vol. 11, no. 7, pp. 447-452, 2017.

[16] P. L. Mennea et al., "Modular linear optical circuits," Optica, vol. 5, no. 9, p. 1087, 2018.

[17] C. Taballione et al., " $8 \times 8$ Programmable Quantum Photonic Processor Based on Silicon Nitride Waveguides," Opt. InfoBase Conf. Pap., vol. Part F114-, no. 19, pp. 22-24, 2018.

A demonstration of an $8 \times 8$ forward-only programmable linear circuit in silicon nitride, benefiting from the notably low optical losses of this material platform, which makes it attractive for linear quantum operations on single photons.

[18] D. Perez et al., "Silicon Photonics Rectangular Universal Interferometer," Laser Photonics Rev., vol. 11, no. 6, 2017.

[19] Y. Xie et al., "Programmable optical processor chips: Toward photonic RF filters with DSP-level flexibility and MHz-band selectivity," Nanophotonics, vol. 7, no. 2, pp. 421-454, 2017.

A comprehensive overview of the various ways a programmable photonic circuit can be used to process microwave signals, and how this type of circuits is transitioning from custom ASPICs to generic programmable PICs. 
[20] T. J. Hall and M. Hasan, "Universal discrete Fourier optics RF photonic integrated circuit architecture," Opt. Express, vol. 24, no. 7, p. 7600, 2016.

[21] I. V. Dyakonov et al., "Reconfigurable Photonics on a Glass Chip," Phys. Rev. Appl., vol. 10, no. 4, p. 1, 2018.

[22] F. Shokraneh, S. Geoffroy-Gagnon, M. S. Nezami, and O. Liboiron-Ladouceur, "A Single Layer Neural Network Implemented by a $4 \times 4$ MZI-Based Optical Processor," IEEE Photonics J., vol. 11, no. 6 , 2019.

[23] L. Lu, L. Zhou, and J. Chen, "Programmable SCOW mesh silicon photonic processor for linear unitary operator," Micromachines, vol. 10, no. 10, 2019.

[24] X. Qiang et al., "Large-scale silicon quantum photonics implementing arbitrary two-qubit processing," Nat. Photonics, vol. 12, no. 9, pp. 534-539, 2018.

[25] J. Wang et al., "Multidimensional quantum entanglement with large-scale integrated optics," vol. 7053, no. March, pp. 1-14, 2018.

[26] C. Schaeff, R. Polster, M. Huber, S. Ramelow, and A. Zeilinger, "Experimental access to higher-dimensional entangled quantum systems using integrated optics," Optica, vol. 2, no. 6, p. 523, 2015.

[27] P. J. Shadbolt et al., "Generating, manipulating and measuring entanglement and mixture with a reconfigurable photonic circuit," Nat. Photonics, vol. 6, no. 1, pp. 45-49, 2012.

[28] D. A. B. Miller, "Waves, modes, communications, and optics: a tutorial," Adv. Opt. Photonics, vol. 11, no. 3, p. 679, 2019.

[29] D. A. B. Miller, "Self-aligning universal beam coupler.," Opt. Express, vol. 21, no. 5, pp. 6360-70, 2013.

[30] D. A. B. Miller, "Perfect optics with imperfect components," Optica, vol. 2, no. 8, pp. 747-750, Aug. 2015.

[31] A. Annoni et al., "Unscrambling light - automatically undoing strong mixing between modes," Light Sci. Appl., no. February, pp. 110, 2017.

Early demonstration of a forward-only programmable mesh to unmix different modes in a waveguide, making use of integrated transparent detectors that measure the intensity of light in the waveguide without inducing additional optical loss.

[32] S. Pai et al., "Parallel fault-tolerant programming of an arbitrary feedforward photonic network," IEEE J. Sel. Top. Quantum Electron. vol. 25, paper 6100813, 2020.

[33] M. Reck, A. Zeilinger, H. J. Bernstein, and P. Bertani, "Experimental realization of any discrete unitary operator," Phys. Rev. Lett., vol. 73, no. 1, pp. 58-61, Jul. 1994.

[34] M. Wang, A. R. Alves, Y. Xing, and W. Bogaerts, "Tolerant, Broadband Tunable $2 \times 2$ Coupler Circuit," Opt. Express, vol. 28, no. 4, pp. 5555-5566, 2020.

[35] D. Pérez-López, A. M. Gutierrez, E. Sánchez, P. DasMahapatra, and J. Capmany, "Integrated photonic tunable basic units using dual- drive directional couplers," Opt. Express, vol. 27, no. 26, p. 38071 , 2019.

[36] K. Choutagunta, I. Roberts, D. A. B. Miller, and J. M. Kahn, "Adapting Mach-Zehnder Mesh Equalizers in Direct-Detection ModeDivision-Multiplexed Links," vol. 38, no. 4, pp. 723-735, 2020.

[37] D. A. B. Miller, "Analyzing and generating multimode optical fields using self-configuring networks," Optica, vol. 7, pp. 794-801, 2020.

[38] J.-F. Morizur et al., "Programmable unitary spatial mode manipulation," J. Opt. Soc. Am. A, vol. 27, no. 11, p. 2524, 2010.

[39] G. Labroille, B. Denolle, P. Jian, P. Genevaux, N. Treps, and J. F. Morizur, "Efficient and mode selective spatial mode multiplexer based on multi-plane light conversion," Opt. Express, vol. 22, no. 13, pp. 488496, 2014.

[40] R. Tanomura, R. Tang, S. Ghosh, T. Tanemura, and T. Nakano, "Robust Integrated Optical Unitary Converter Using Multiport Directional Couplers," J. Light. Technol., vol. 38, no. 1, pp. 60-66, 2020.

[41] D. A. B. Miller, "Setting up meshes of interferometers - reversed local light interference method," Opt. Express, vol. 25, no. 23, p. 29233, 2017.

[42] H. W. Li et al., "Calibration and high fidelity measurement of a quantum photonic chip," New J. Phys., vol. 15, 2013.

[43] G. Cong et al., "Arbitrary reconfiguration of universal silicon photonic circuits by bacteria foraging algorithm to achieve reconfigurable photonic digital-to-analog conversion," Opt. Express, vol. 27, no. 18, p. 24914, 2019.

[44] D. Pérez et al., "Multipurpose silicon photonics signal processor core," Nat. Commun., vol. 8, no. 1, pp. 1-9, 2017.

The first experimental demonstration of a recirculating waveguide mesh with 7 unit cells that can be programmed to perform more than hundred different functions.

[45] D. Pérez, I. Gasulla, and J. Capmany, "Field-programmable photonic arrays," Opt. Express, vol. 26, no. 21, p. 27265, 2018.

[46] A. Rahim, T. Spuesens, R. Baets, and W. Bogaerts, "Open-Access Silicon Photonics: Current Status and Emerging Initiatives," Proc. IEEE, vol. 106, no. 12, pp. 2313-2330, 2018.

[47] P. Munoz et al., "Foundry Developments Toward Silicon Nitride Photonics from Visible to the Mid-Infrared," IEEE J. Sel. Top. Quantum Electron., vol. 25, no. 5, 2019.

[48] M. Teng et al., "Miniaturized Silicon Photonics Devices for Integrated Optical Signal Processors," J. Light. Technol., vol. 38, no. 1, pp. 6-17, 2020.

[49] W. D. Sacher et al., "Monolithically Integrated Multilayer Silicon Nitride-on-Silicon Waveguide Platforms for 3-D Photonic Circuits and Devices," Proc. IEEE, vol. 106, no. 12, pp. 2232-2245, 2018. 
[50] C. Baudot et al., "Developments in $300 \mathrm{~mm}$ silicon photonics using traditional CMOS fabrication methods and materials," 2017 IEEE Int. Electron Devices Meet., pp. 765-768, 2017.

[51] N. M. Fahrenkopf, C. McDonough, G. L. Leake, Z. Su, E. Timurdogan, and D. D. Coolbaugh, "The AIM Photonics MPW: A Highly Accessible Cutting Edge Technology for Rapid Prototyping of Photonic Integrated Circuits," IEEE J. Sel. Top. Quantum Electron., vol. 25, no. 5, pp. 1-6, 2019.

[52] J. Chiles, S. Buckley, N. Nader, S. W. Nam, R. P. Mirin, and J. M. Shainline, "Multi-planar amorphous silicon photonics with compact interplanar couplers, cross talk mitigation, and low crossing loss," APL Photonics, vol. 2, no. 11, 2017.

[53] J. Van Campenhout, W. M. J. Green, S. Assefa, and Y. A. Vlasov, "Integrated NiSi waveguide heaters for CMOS-compatible silicon thermo-optic devices.," Opt. Lett., vol. 35, no. 7, pp. 1013-1015, 2010.

[54] A. Masood et al., "Comparison of heater architectures for thermal control of silicon photonic circuits," in Proceedings of the 10th International Conference on Group IV Photonics, 2013, p. ThC2.

[55] M. Milanizadeh, D. Aguiar, A. Melloni, and F. Morichetti, "Canceling Thermal Cross-Talk Effects in Photonic Integrated Circuits," J. Light. Technol., vol. 37, no. 4, pp. 1325-1332, 2019.

[56] R. A. Soref and B. R. Bennett, "Electrooptical effects in silicon," IEEE J. Quantum Electron., vol. 23, no. 1, pp. 123-129, 1987.

[57] G. T. Reed, G. Mashanovich, F. Y. Gardes, and D. J. Thomson, "Silicon optical modulators," Nat. Photonics, vol. 4, no. 8, pp. 518-526, 2010.

[58] F. A. Memon et al., "Silicon Oxycarbide Platform for Integrated Photonics," J. Light. Technol., vol. 38, no. 4, pp. 784-791, 2020.

[59] W. Jin, R. G. Polcawich, P. A. Morton, and J. E. Bowers, "Piezoelectrically tuned silicon nitride ring resonator," Opt. Express, vol. 26, no. 3, pp. 3174-3187, 2018.

[60] N. Hosseini et al., "Stress-optic modulator in TriPleX platform using a piezoelectric lead zirconate titanate (PZT) thin film," Opt. Express, vol. 23, no. 11, p. 14018, 2015.

[61] W. De Cort, J. Beeckman, T. Claes, K. Neyts, and R. Baets, "Wide tuning of silicon-on-insulator ring resonators with a liquid crystal cladding," Opt. Lett., vol. 36, no. 19, pp. 3876-3878, Oct. 2011.

[62] Y. Xing et al., "Digitally Controlled Phase Shifter Using an SOI Slot Waveguide With Liquid Crystal Infiltration," Photon. Technol. Lett., vol. 27, no. 12, pp. 1269-1272, Jun. 2015.

[63] S. Abel et al., "Large Pockels effect in micro- and nanostructured barium titanate integrated on silicon," Nat. Mater., vol. 18, no. 1, pp. 42-47, 2019.

[64] B. Desiatov, A. Shams-Ansari, M. Zhang, C. Wang, and M. Lončar, "Ultra-low-loss integrated visible photonics using thin-film lithium niobate," Optica, vol. 6, no. 3, p. 380, 2019.

[65] K. Alexander et al., "Nanophotonic Pockels modulators on a silicon nitride platform," Nat. Commun., vol. 9, no. 1, pp. 4-9, 2018.
[66] J. Leuthold et al., "Silicon-Organic hybrid electro-optical devices," IEEE J. Sel. Top. Quantum Electron., vol. 19, no. 6, pp. 114126, Nov. 2013.

[67] C. Errando-Herranz, A. Y. Takabayashi, P. Edinger, H. Sattari, K. B. Gylfason, and N. Quack, "MEMS for Photonic Integrated Circuits," IEEE J. Sel. Top. Quantum Electron., vol. 26, no. 2, pp. 1-1, 2019.

[68] N. Quack et al., "MEMS-enabled Silicon Photonic Integrated Devices and Circuits," IEEE J. Quantum Electron., vol. 56, no. 1, p. 8400210, 2020.

[69] C. Hoessbacher et al., "The plasmonic memristor: a latching optical switch," Optica, vol. 1, no. 4, p. 198, 2014.

[70] C. Rios et al., "Integrated all-photonic non-volatile multi-level memory," Nat. Photonics, vol. 9, no. 11, pp. 725-732, 2015.

[71] M. Wuttig, H. Bhaskaran, and T. Taubner, "Phase-change materials for non-volatile photonic applications," Nat. Photonics, vol. 11, no. 8, pp. 465-476, 2017.

[72] F. Morichetti et al., "Non-invasive on-chip light observation by contactless waveguide conductivity monitoring," IEEE J. Sel. Top. Quantum Electron., vol. 20, no. 4, pp. 292-301, 2014.

[73] H. Jayatilleka, H. Shoman, L. Chrostowski, and S. Shekhar, "Photoconductive heaters enable control of large-scale silicon photonic ring resonator circuits," Optica, vol. 6, no. 1, pp. 84-91, 2019.

[74] S. Grillanda et al., "Non-invasive monitoring and control in silicon photonics using CMOS integrated electronics," Optica, vol. 1, no. 3, p. 129, 2014.

[75] A. Annoni et al., "Automated routing and control of silicon photonic switch fabrics," IEEE J. Sel. Top. Quantum Electron., vol. 22, no. 6, pp. 4-11, 2016.

[76] P. Dumais et al., "Silicon Photonic Switch Subsystem with 900 Monolithically Integrated Calibration Photodiodes and 64-Fiber Package," J. Light. Technol., vol. 36, no. 2, pp. 233-238, 2018.

[77] H. Chen, X. Luo, and A. W. Poon, "Cavity-enhanced photocurrent generation by $1.55 \mu \mathrm{m}$ wavelengths linear absorption in a $\mathrm{p}$-i-n diode embedded silicon microring resonator," Appl. Phys. Lett., vol. 95, no. 17, 2009.

[78] A. Ribeiro and W. Bogaerts, "Digitally controlled multiplexed silicon photonics phase shifter using heaters with integrated diodes," Opt. Express, vol. 25, no. 24, p. 29778, 2017.

[79] L. Zimmermann et al., "BiCMOS Silicon Photonics Platform," in Optical Fiber Communication Conference, 2015, p. Th4E.5.

[80] J. S. Orcutt et al., "Nanophotonic integration in state-of-the-art CMOS foundries," Opt. Express, vol. 19, no. 3, pp. 2335-2346, Jan. 2011.

[81] V. Stojanović et al., "Monolithic silicon-photonic platforms in state-of-the-art CMOS SOI processes [Invited]," Opt. Express, vol. 26, no. 10, p. 13106, 2018. 
[82] L. Carroll et al., "Photonic packaging: Transforming silicon photonic integrated circuits into photonic devices," Appl. Sci., vol. 6, no. 12 , pp. 1-21, 2016

[83] D. Patterson, I. De Sousa, and L.-M. Archard, "The future of packaging with silicon photonics," Chip Scale Rev., vol. 21, no. 1, pp. 110, 2017.

[84] A. Ribeiro, S. Declercq, U. Khan, M. Wang, and L. Van Iseghem, "Column-row addressing of thermo-optic phase shifters for controlling large silicon photonic circuits," J. Sel. Top. Quantum Electron., vol. 15, pp. 1-8, 2020.

[85] M. Pantouvaki et al., "Active Components for $50 \mathrm{~Gb} / \mathrm{s}$ NRZ-OOK Optical Interconnects in a Silicon Photonics Platform," J. Light. Technol., vol. 35, no. 4, pp. 631-638, 2017.

[86] H. Chen et al., "100-Gbps RZ Data Reception in 67-GHz SiContacted Germanium Waveguide p-i-n Photodetectors," J. Light. Technol., vol. 35, no. 4, pp. 722-726, Feb. 2017.

[87] D. Pérez, I. Gasulla, and J. Capmany, "Toward Programmable Microwave Photonics Processors," J. Light. Technol., vol. 36, no. 2, pp. 519-532, 2018.

[88] M. Zoldak, L. Halmo, J. P. Turkiewicz, S. Schumann, and R. Henker, "Packaging of ultra-high speed optical fiber data interconnects," Opt. Fibers Their Appl. 2017, vol. 10325, no. February 2017, p. 103250R, 2017.

[89] A. E. Willner, S. Khaleghi, M. R. Chitgarha, and O. F. Yilmaz, "Alloptical signal processing," J. Light. Technol., vol. 32, no. 4, pp. 660-680, Feb. 2014

[90] J. M. Ramirez et al., "III-V-on-Silicon Integration: From Hybrid Devices to Heterogeneous Photonic Integrated Circuits," IEEE J. Sel. Top. Quantum Electron., vol. 26, no. 2, 2020.

[91] A. Y. Liu and J. Bowers, "Photonic integration with epitaxial III-V on silicon," IEEE J. Sel. Top. Quantum Electron., vol. 24, no. 6, 2018.

[92] J. Zhang et al., "Transfer-printing-based integration of a III-V-onsilicon distributed feedback laser," Opt. Express, vol. 26, no. 7, pp. 8821-8830, 2018.

[93] T. Thiessen, J. C. C. Mak, J. Da Fonseca, K. Ribaud, C. Jany, and J. K. S. Poon, "Back-Side-on-BOX Heterogeneously Integrated III-V-onSilicon O-Band Distributed Feedback Lasers," J. Light. Technol., no. c, pp. 1-8, 2020.

[94] A. Lopez, D. Perez, P. DasMahapatra, and J. Capmany, "Autorouting algorithm for field-programmable photonic gate arrays," Opt. Express, vol. 28, no. 1, pp. 737-752, 2020.

[95] X. Chen, P. Stroobant, M. Pickavet, and W. Bogaerts, "Graph Representations for Programmable Photonic Circuits," J. Light. Technol., vol. 38, 2020.

[96] I. Zand and W. Bogaerts, "Effects of Coupling and Phase Imperfections in Programmable Photonic Hexagonal Waveguide Meshes," Photonics Res., vol. 8, no. 2, pp. 211-218, 2020.
[97] W. Bogaerts and A. Rahim, "Programmable Photonics: An Opportunity for an Accessible Large-Volume PIC Ecosystem," IEEE J. Sel. Top. Quantum Electron., vol. 26, no. 5, 2020.

An simple techno-economic analysis of how general purpose programmable photonic circuits can reduce the cost of prototyping new photonics applications.

[98] D. Novak et al., "Radio-over-fiber technologies for emerging wireless systems," IEEE J. Quantum Electron., vol. 52, no. 1, pp. 1-11, 2016.

[99] M. Dubrovsky, M. Ball, and B. Penkovsky, "Optical Proof of Work," https://arxiv.org/abs/1911.05193, 2019.

[100] Y. Paquot, J. Schroeder, M. D. Pelusi, and B. J. Eggleton, "Alloptical hash code generation and verification for low latency communications," Opt. Express, vol. 21, no. 20, pp. 3-5, 2013.

[101] J. Wang, F. Sciarrino, A. Laing, and M. G. Thompson, "Integrated photonic quantum technologies," Nat. Photonics, 2019.

[102] E. J. Norberg, R. S. Guzzon, J. S. Parker, L. A. Johansson, and L. A. Coldren, "Programmable photonic microwave filters monolithically integrated in InP-InGaAsP," J. Light. Technol., vol. 29, no. 11, pp. 16111619, 2011.

[103] J. Wang et al., "Reconfigurable radio-frequency arbitrary waveforms synthesized in a silicon photonic chip," Nat. Commun., vol. 6, pp. 1-8, 2015.

[104] M. Burla et al., “On-chip CMOS compatible reconfigurable optical delay line with separate carrier tuning for microwave photonic signal processing," Opt. Express, vol. 19, no. 22, p. 21475, 2011.

[105] L. Liu et al., "Photonic measurement of microwave frequency using a silicon microdisk resonator," Opt. Commun., vol. 335, pp. 266 270, 2015.

[106] D. Perez-Lopez, E. Sanchez, and J. Capmany, "Programmable True-Time Delay Lines using integrated waveguide meshes," J. Light. Technol., 2018.

[107] B. Behroozpour, P. A. M. Sandborn, M. C. Wu, and B. E. Boser, "Lidar System Architectures and Circuits," IEEE Commun. Mag., vol. 55, no. 10, pp. 135-142, 2017.

[108] M. J. R. Heck, "Highly integrated optical phased arrays: Photonic integrated circuits for optical beam shaping and beam steering," Nanophotonics, vol. 6, no. 1, pp. 93-107, 2017.

[109] K. Van Acoleyen, "Efficient Light Collection and Direction-ofArrival Estimation Using a Photonic Integrated Circuit," Photonics ..., vol. 24, no. 11, pp. 933-935, 2012.

[110] D. A. B. Miller, "Establishing optimal wave communication channels automatically," J. Light. Technol., vol. 31, no. 24, pp. 39873994, 2013.

[111] E. Luan, H. Shoman, D. M. Ratner, K. C. Cheung, and L. Chrostowski, "Silicon photonic biosensors using label-free detection," Sensors, vol. 18, no. 10, pp. 1-42, 2018. 
[112] A. Z. Subramanian et al., "Silicon and silicon nitride photonic circuits for spectroscopic sensing on-a-chip [Invited]," Photonics Res., vol. 3, no. 5, pp. B47-B59, Oct. 2015.

[113] Y. Li et al., "Six-beam homodyne laser Doppler vibrometry based on silicon photonics technology," Opt. Express, vol. 26, no. 3, p. 3638, 2018.

[114] S. M. Trimberger, "Three ages of FPGAs: A retrospective on the first thirty years of FPGA technology," Proc. IEEE, vol. 103, no. 3, pp. 318-331, Mar. 2015.

[115] I. Mohomed and P. Dutta, "The Age of DIY and Dawn of the Maker Movement," ACM SIGMOBILE Mob. Comput. Commun. Rev., vol. 18, no. 4, pp. 41-43, 2015.

[116] J. Preskill, "Quantum Computing in the NISQ era and beyond," Quantum, vol. 7, p.79, 2018.
[117] F. Arute et al., "Quantum supremacy using a programmable superconducting processor," Nature, vol. 574, no. 7779, pp. 505-510, 2019.

[118] J. Biamonte, P. Wittek, N. Pancotti, P. Rebentrost, N. Wiebe, and S. Lloyd, "Quantum machine learning," Nature, vol. 549, no. 7671, pp. 195-202, 2017.

[119] G. R. Steinbrecher, J. P. Olson, D. Englund, and J. Carolan, "Quantum optical neural networks," npj Quantum Inf., vol. 5, no. 1, pp. 1-9, 2019.

[120] F. M. Miatto, M. Epping, and N. Lütkenhaus, "Hamiltonians for one-way quantum repeaters," Quantum, vol. 2, no. 1, p. 75, 2018. 